\title{
BARRIER RF SYSTEM AND APPLICATIONS IN MAIN INJECTOR*
}

\author{
W. Chou ${ }^{\dagger}$ and D. Wildman, Fermilab, Batavia, IL 60510, U.S.A. \\ A. Takagi, KEK, Tsukuba, Japan, H. Zheng, Caltech, Pasadena, CA 91125, U.S.A.
}

\section{Abstract}

A novel broadband RF system - the barrier RF - has been designed, fabricated and installed in the Fermilab Main Injector (MI). It uses nanocrystal magnetic alloy called Finemet and high voltage fast MOSFET switches. The system delivers $\pm 10 \mathrm{kV}$ square pulses at $90 \mathrm{kHz}$. It can stack two proton batches injected from the Booster and squeeze them into the size of one so that the bunch intensity is increased. The high intensity beams have been successfully accelerated to $120 \mathrm{GeV}$ with small losses. The problem of large longitudinal emittance growth is under investigation. A second system will be installed during the fall shutdown and be tested for the so-called fast stacking scheme to continuously stack up to 12 Booster batches in the MI. This system is also used for cleaning up the leaking-out dc beams from slip stacking to reduce beam loss. This work is part of the US-Japan collaborative agreement.

\section{INTRODUCTION}

In order to increase the luminosity of the Tevatron collider program Run2 and protons-on-target of the neutrino program NuMI, a key is to increase the proton intensity in the Main Injector. Presently the bottleneck in the Fermilab accelerator complex is the Booster, which is a 30 years old machine and can only deliver about 5E12 protons per cycle. The Main Injector, on the other hand, is a new one and capable to accelerate much more protons than what the Booster can deliver. One solution to solve this problem is by stacking, i.e., to stack Booster beams in the Main Injector. The MI acceptance (about $0.7 \mathrm{eV}-\mathrm{s}$ ) is much larger than the Booster beam emittance (about 0.13 eV-s). This makes it possible to stack two Booster bunches in one MI rf bucket. There are different methods to implement this scheme. One is slip stacking as described elsewhere [1]. This paper introduces another method called barrier rf stacking. The basic idea is as follows: Let the injected beam debunch, then use rf barriers to manipulate the continuous beam so that the total beam size would change. The manipulation can be done either by adiabatic squeezing or by reflecting and folding. The latter requires two barrier rf systems and is called fast stacking because of its continuous manner. At this moment one barrier rf system has been installed in the MI for adiabatic stacking as well as for cleaning the leaking beams from slip stacking. A second system is under construction and will be installed during this fall shutdown.

\footnotetext{
*Work supported by Universities Research Association, Inc. under contract No. DE-AC02-76CH03000 with the U.S. Dept. of Energy. †chou@fnal.gov
}

\section{BARRIER RF SYSTEM}

The barrier rf system specs are listed in Table 1. It consists of a cavity (Figure 1) and a modulator (Figure 2). The cavity is made of seven Finemet cores. Finemet is a nanocrystal magnetic alloy patented by Hitachi. It has high magnetic permeability and low $\mathrm{Q}$ in the $\mathrm{MHz}$ range and is especially suitable for our application. The main components of the modulator are a pair of bipolar high voltage fast MOSFET switches made by Behlke Co. in Germany. This system is similar to an $\mathrm{rf}$ chopper used at HIMAC in Japan [2-5].

Table 1: Barrier rf system parameters

\begin{tabular}{|l|l|}
\hline Pulse peak voltage & $\pm 10 \mathrm{kV}$ \\
\hline Pulse maximum length & $0.5 \mu \mathrm{s}$ \\
\hline Gap between pulses & $0.4-11 \mu \mathrm{s}$ \\
\hline Pulse repetition rate & $90 \mathrm{kHz}$ \\
\hline Burst length & $400 \mathrm{~ms}$ \\
\hline Burst repetition rate & $0.5 \mathrm{~Hz}$ \\
\hline
\end{tabular}

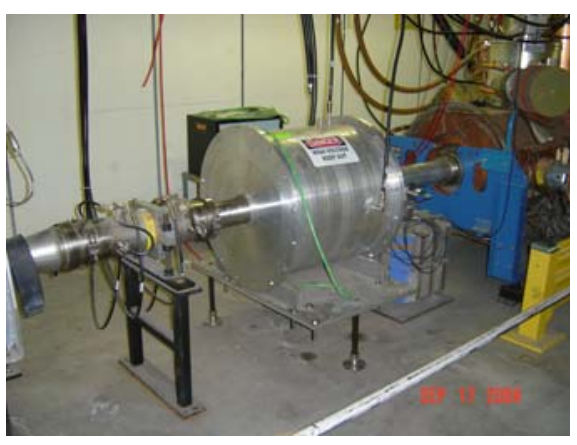

Figure 1: A barrier rf cavity in the Main Injector tunnel.

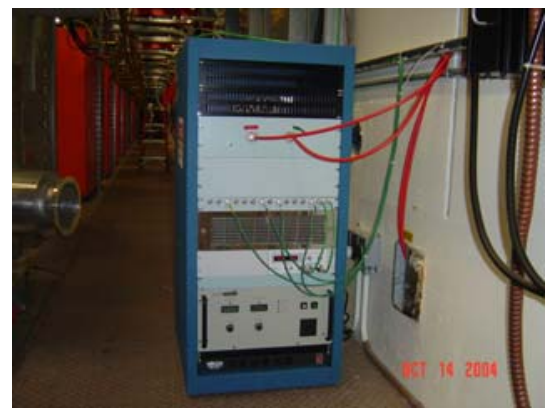

Figure 2: A barrier rf modulator in MI-60 bulding. 


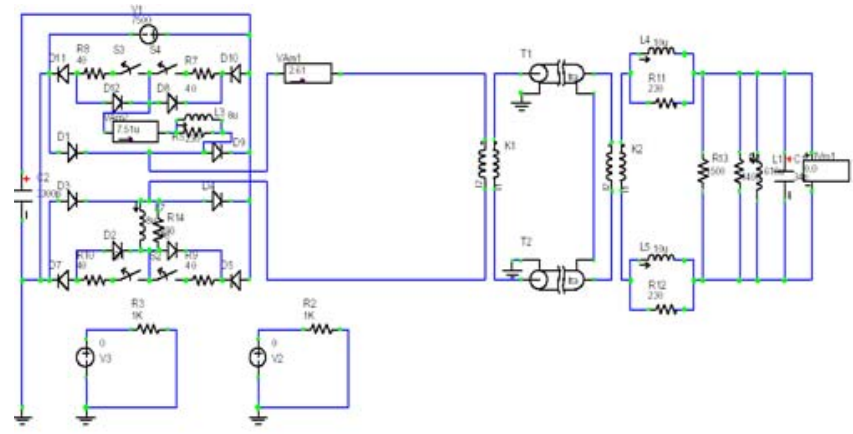

Figure 3: SPICE model of the circuit for the barrier rf power supply.

Figure 3 shows the circuit diagram. A particular concern in the design is radiation damage to the switches. The cavity is an inductive load, which would generate "ringing" after a single pulse. In order to minimize the ringing, the switches should be close to the cavity. However, the radiation level in the tunnel is high during machine operation, which could destroy the switches in a short time. A solution was found by using two transformers, one between the switches and the cable, another between the cable and the cavity. This solves the impedance mismatch problem when long cables are used.

\section{BEAM EXPERIMENTS}

\section{Adiabatic stacking}

Two Booster batches, each containing 84 proton bunches, are injected into the MI consecutively and captured by the main rf system at $53 \mathrm{MHz}$. The total beam size is $3.2 \mu \mathrm{s}$. This beam is debunched and confined by $\mathrm{rf}$ barriers. The barriers are then moved towards each other and compress the beam to half size, namely, $1.6 \mu \mathrm{s}$. The compressed beam is recaptured by the $53 \mathrm{MHz}$ rf and accelerated to $120 \mathrm{GeV}$. The process is shown in Figure 4.

Figure 5 is an oscilloscope plot showing the movement of the barriers (pink curve) and compression of the beam (blue curve). Figure 6 is a mountain view, in which one can see clearly how a long beam is squeezed into a short one by moving barriers.

From Figure 4, it is seen the main loss is during initial acceleration when the rf bucket is shrinking. However, there is no loss observed during transition crossing. The beam emittance measured right before the transition is $0.73 \mathrm{eV}-\mathrm{s}$, which is a measure of the machine acceptance at transition. The beam intensity at $120 \mathrm{GeV}$ reaches $8.3 \mathrm{E} 12$, about $60 \%$ higher than without stacking.

The large emittance of the stacked beam is a concern of the pbar production, but should be ok for NuMI operation. The source of such a large emittance dilution (from 0.13 to $0.73 \mathrm{eV}-\mathrm{s}$ ) is under investigation. One possible explanation is the instability of the debunched beam.

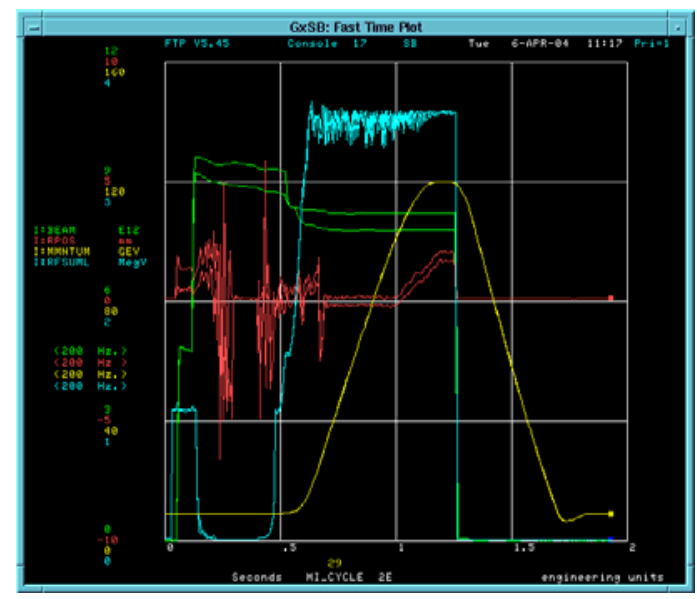

Figure 4: Main Injector cycle. Yellow is the energy ramp curve; blue the $53 \mathrm{MHz}$ rf curve, which shows the five stages: capture - debunch - squeeze (near zero voltage) - recapture - acceleration; green the beam intensity (two Booster injections).

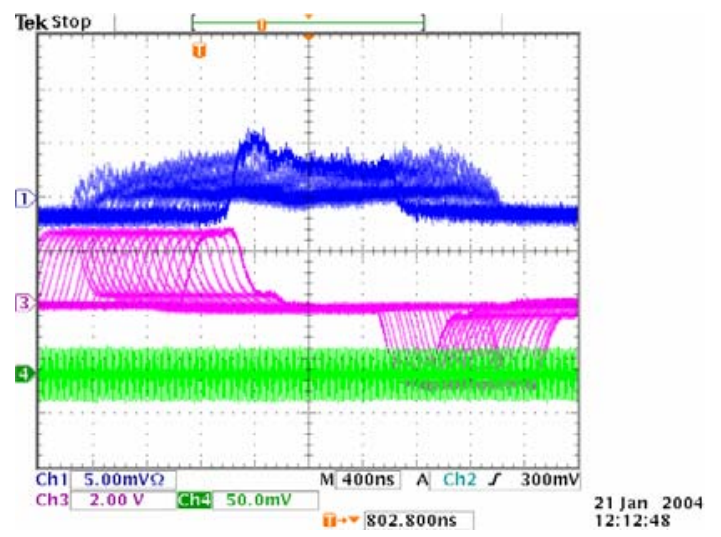

Figure 5: Dynamic plot of barrier rf voltage (middle curve in purple) and beam (top curve in blue). When the barriers move in, the beam gets squeezed.

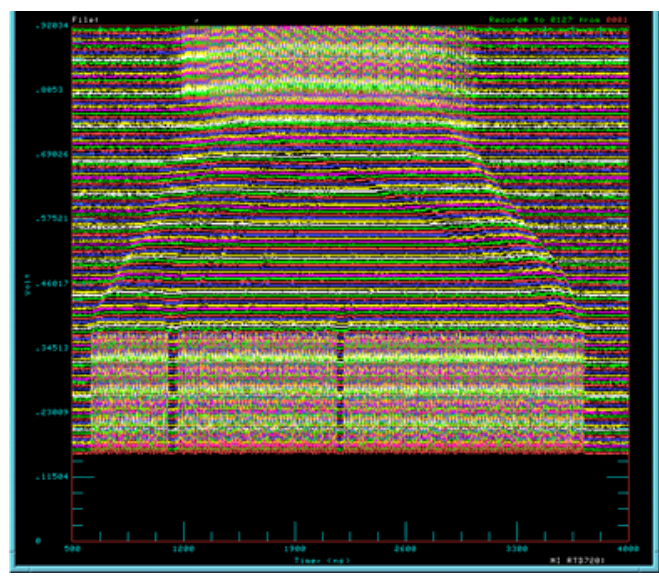

Figure 6: Mountain view picture of the beam. The vertical axis is time. The beam is squeezed to half size by moving the rf barriers. 


\section{Injection gap cleaning}

A recent application of the barrier rf is to clean up the gap between the slip-stacked batch and the NuMI batches. Due to mismatch between Booster bunch and slip stacking rf bucket, a considerable amount of beams (about $10 \%)$ are not captured during slip stacking and become dc beams, which move at a rate of $\eta \times \Delta p / p$ (slip factor times momentum spread) towards the unoccupied part of the machine. For a particle of an energy error of $15 \mathrm{MeV}$, the moving speed is about $1 \mu \mathrm{s}$ (about $10 \%$ of the machine size) in one Booster cycle $(67 \mathrm{~ms})$. When a series of 5 NuMI batches are injected, these dc beams could spread to everywhere and be kicked to the downstream quads (Q104 and Q105) by injection kickers and cause high radiation activation. This problem can be avoided by using barrier $\mathrm{rf}$ to prevent the dc beams from leaking. Figure 7 is a comparison of the beam loss at Q104 with and without $\mathrm{rf}$ barrier. It is obvious that the gap when injecting the first 3 NuMI batches is much cleaner. However, the effect on the last 2 batches is minimal, which is to be investigated.

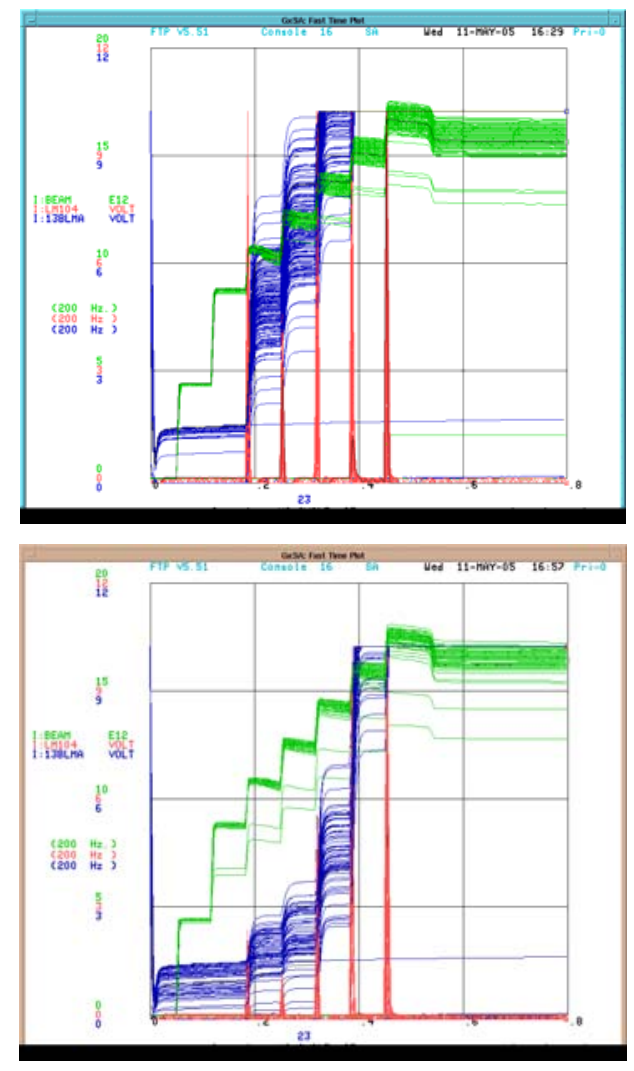

Figure 7: Green curves are 7 Booster batches injected into the MI. The first two are slip stacked for pbar production, the next five for NuMI. Red curves are instantaneous losses at quad Q104 (downstream from the injection kicker), blue curves the integrated loss. Top: without rf barrier; bottom: with rf barrier. It is seen that the losses when injecting the first three NuMI batches are considerably lower in the bottom plot.

\section{FUTURE EXPERIMENT}

A second barrier rf system will be installed in the MI this fall. With two systems, it will be possible to implement the fast stacking scheme. This scheme was first proposed by Griffin [6] and extensively analyzed by $\mathrm{Ng}$ [7]. It uses one stationary (or slowly moving) barrier and a fast moving barrier. The injected beam would have an energy offset and the injection would be continuous. The beams would be reflected and folded by the barriers so that the earlier injected beams would be moved and repositioned on top of the later ones in the longitudinal phase space. Simulation shows the MI would be able to accept 12 Booster batches instead of 6 as it does now $[7,8]$. A key issue is to keep the Booster beam energy spread small (preferred to be less than $\pm 10 \mathrm{MeV}$ after bunch rotation). A powerful low level rf control system will also be needed.

The goal of this experiment is to double the NuMI beam intensity from $3 \mathrm{E} 12$ to $6 \mathrm{E} 12$.

\section{ACKNOWLEDGEMENT}

This work started several years ago and is the result of a number of people's contribution at different stages, including J. Griffin, J. MacLachlan, K-Y. Ng, J-F. Ostiguy, C. Ankenbrandt, C. Bhat, I. Kourbanis, Y. Mori, A. Marchionni, D. Johnson and D. Michael. The authors would like to express their gratitude to these colleagues.

This work is partially funded by the US-Japan collaboration on high intensity proton facility R\&D.

\section{REFERENCES}

[1] K. Seiya, this conference.

[2] W. Chou et al., "Design and Measurements of a Pulsed Beam Transformer as a Chopper," KEK Report 98-10 (September 1998).

[3] W. Chou et al., Proc. 1999 PAC (New York, USA), p. 565.

[4] Y. Shirakabe et al., Proc. 2000 EPAC (Vienna, Austria), p. 2468.

[5] C. Ohmori et al., NIM A 526, 215-221 (2004).

[6] http://www-bd.fnal.gov/pdriver/barrier/pdf/griffin.pdf

[7] K.Y. Ng, "Doubling Main Injector Beam Intensity using RF Barriers," AIP Conference Proc. 642, p. 226 (2002); also see FERMILAB-FN-715, FERMILABTM-2183 (2002).

[8] W. Chou et al., Proc. 2003 PAC (Portland, USA), p. 2922. 